Review article

\title{
Mesenchymal stem cell therapy for osteoarthritis
}

\author{
Hassan Afizah ${ }^{\mathrm{a}, \mathrm{b}}$, James Hoi Po Hui ${ }^{\mathrm{a}, \mathrm{b}, *}$

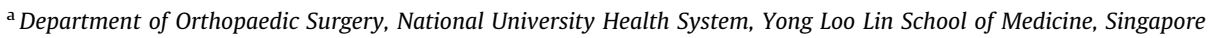 \\ ${ }^{\mathrm{b}}$ Tissue Engineering Programme, Department of Orthopaedic Surgery, Life Sciences Institute, National University of Singapore, Singapore
}

\section{A R T I C L E I N F O}

\section{Article history:}

Received 19 May 2016

Received in revised form 8 June 2016

Accepted 9 June 2016

Available online 22 June 2016

\section{Keywords:}

Stem cell

Osteoarthritis

Infusion

Pain

Dosage

\begin{abstract}
A B S T R A C T
The versatility of mesenchymal stem cells (MSCs) as a treatment modality has landed it another repair target: osteoarthritis, a crippling cartilage disease that frequently afflicts the aged population. Through many studies, this newly discovered method has been shown to significantly alleviate the pain experienced by osteoarthritic patients. Notwithstanding the effectiveness of MSCs in this regard, varying degrees of success rates have also been reported, which is probably attributable to the different approaches adopted in harnessing MSCs' therapeutic value. Accordingly, it is pertinent to understand the contributory factors like MSC type, dosage, size of osteoarthritic lesion, MSC carrier, and mode of infusion, which would be briefly discussed in this review.
\end{abstract}

\section{Introduction}

A PubMed search on the clinical usage of mesenchymal stem cells (MSCs) for osteoarthritis (OA) in January 2016 yielded 18 papers of Level $1,{ }^{1} 2,{ }^{2} 3,,^{3-7} 4,{ }^{8-17} 5^{18}$ clinical trials, with the earliest reported in $2002^{4}$ (Table 1 ). The majority of the published reports are preliminary studies, indicative that investigation of MSC usage for OA is still recent. However, judging from the 50 trials listed in the clinicaltrials.gov database, more are in the works. This carries hope of more breakthrough discoveries.

Pain relief is a common outcome experienced by OA patients who received MSC therapy. There are several groups worldwide which are actively pursuing this interest. Orozco et al. from Spain probed in separate studies, the effect of treating patients diagnosed with grade 2-4 chronic OA with autologous ${ }^{8}$ or allogeneic bone marrow stem cells (BMSCs). ${ }^{1}$ He noted remarkably early analgesic effects of both kinds of MSCs, accompanied with significant functional recovery. Korean research groups have also initiated research in this niche area, with exclusive interest in the usage of adipose-derived MSCs (ADSCs), ${ }^{3,6,7,11,16,17}$ perchance due to the country's famous reputation as a plastic surgery hotspot. Same-day isolated MSCs from adipose tissue of infrapatellar fat pad and

\footnotetext{
* Corresponding author at: Department of Orthopaedic Surgery, National University of Singapore, NUHS Tower Block, Level 11, IE Kent Ridge Road, Singapore 119228, Singapore. Tel.: +65 67724321.

E-mail addresses: dosafiz@nus.edu.sg (H. Afizah), doshuij@nus.edu.sg (J.H.P.Hui).
}

buttocks were injected into the knees of patients suffering up to Kellgren-Lawrence grade 3 OA. Patients enjoyed pain relief even at 2 years after the treatment. Iranian research institutes have also reported their pilot studies. ${ }^{12-15}$ Emadedin et al.'s 6 patients who were afflicted with severe OA received injection of $>20 \times 10^{6}$ BMSCs. Mean VAS pain scores peaked at 6 months, with gradual decline noted within the 12 months of treatment. ${ }^{13}$

As the various groups' methods of exploring MSC efficacy differed to an appreciable extent, this paper sets out to compare based on similar factors, where possible.

\section{Autologous or allogeneic MSCs?}

MSCs' unique stealth capabilities through immunosuppression of natural killer cells and T cells ${ }^{19}$ make them well-suited to play an allogeneic role. Allogeneic MSCs provide an alternative to autologous MSCs possibly already subjected to a damaging environment present in the OA knee. ${ }^{20}$ Among the publications listed above, only one applied allogeneic MSCs for OA treatment. ${ }^{1}$ Allogeneic approaches are still shunned over safety concerns that these cells could transfer infectious diseases. ${ }^{21}$ This pattern could be set to change, as out of 50 listed ongoing clinical trials, 14 are allogeneic in nature. Vega et al. injected passage 2-3 allogeneic BMSCs from 3 healthy human donors in a trial involving 30 patients. Significantly improved pain relief was attained with MSC treatment. Mean algofunctional indices showed that cell treatment led to significantly better outcome in relieving disability. Measurement of effect size, however, revealed that 


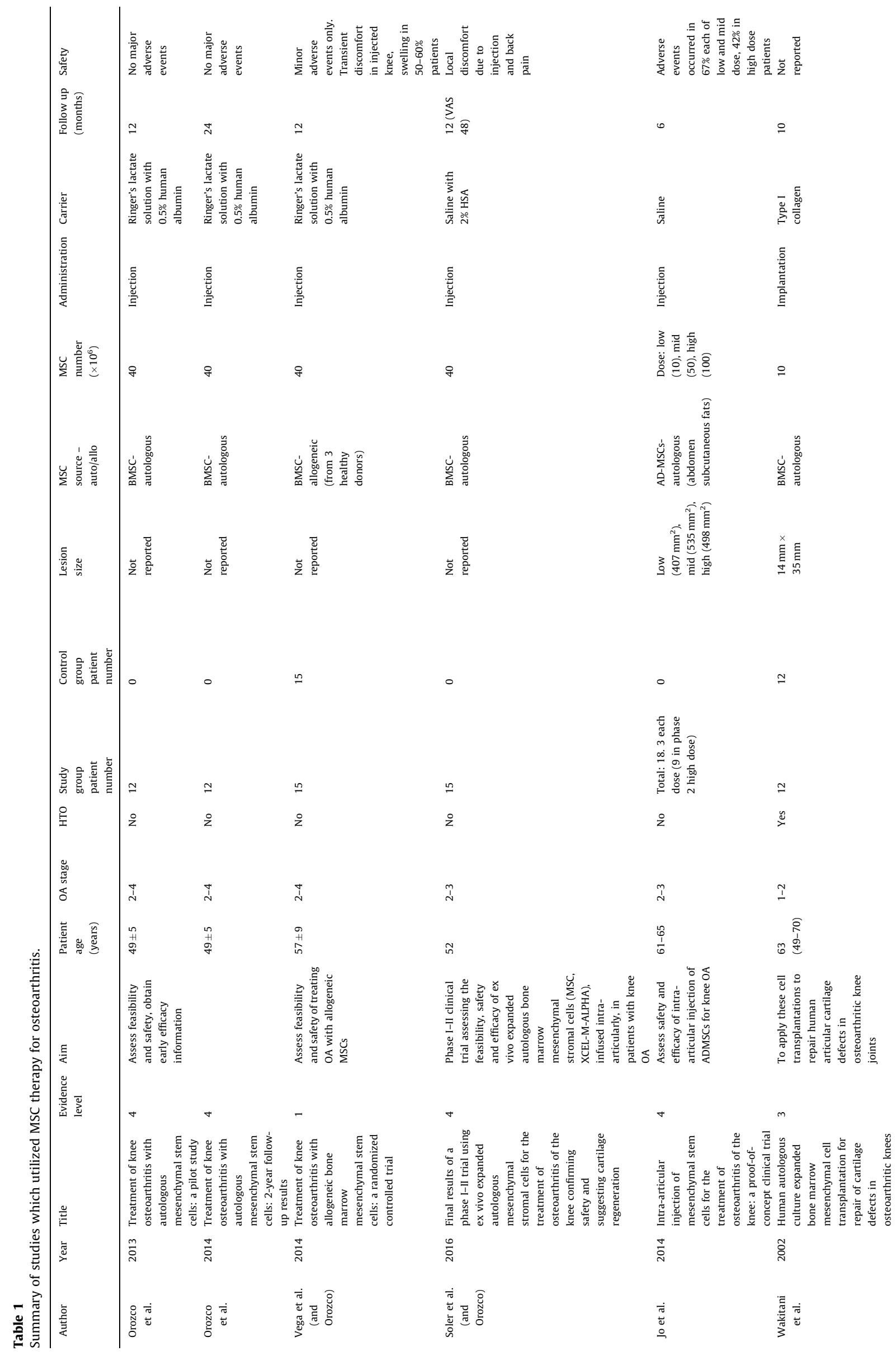



Inmm!n
In
InIIIn!
ซี
言

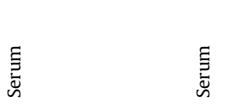

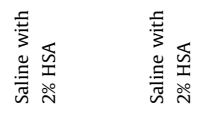
$\simeq$

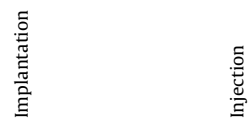

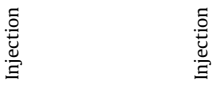

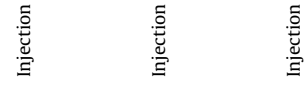
‡
告
总
鵕
m $\quad$
荬

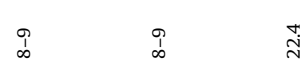
总
$\stackrel{\infty}{\stackrel{\infty}{-}}$

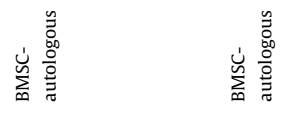

峉高

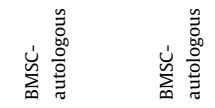

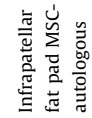

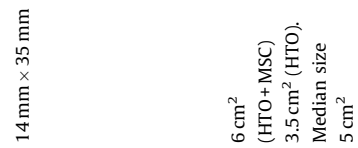

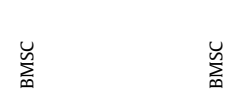

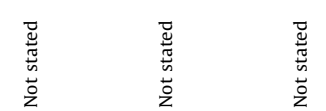

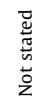

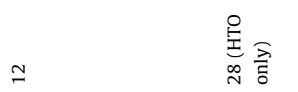

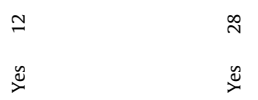

I $\quad$ 产

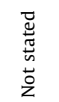

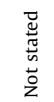

可

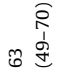<smiles>C1CCCCC1</smiles>

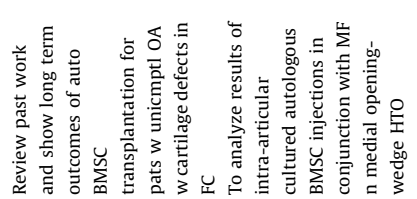

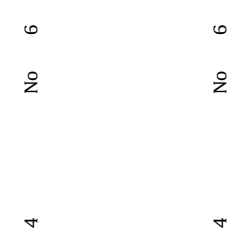

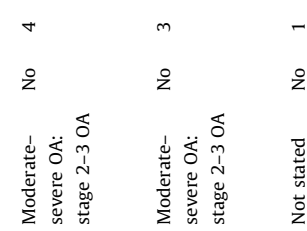

$\stackrel{20}{\sim}$

$\stackrel{\circ}{2}$

ฉ

$\stackrel{2}{2}$

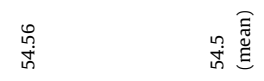

皇

蓄高

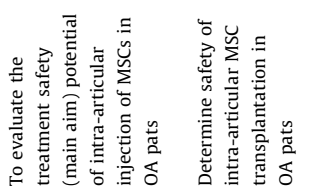

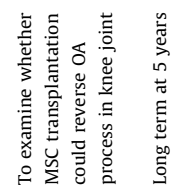

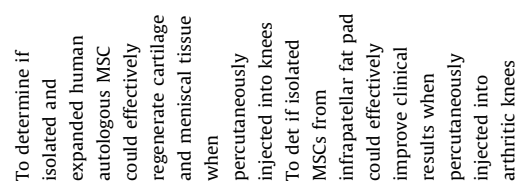

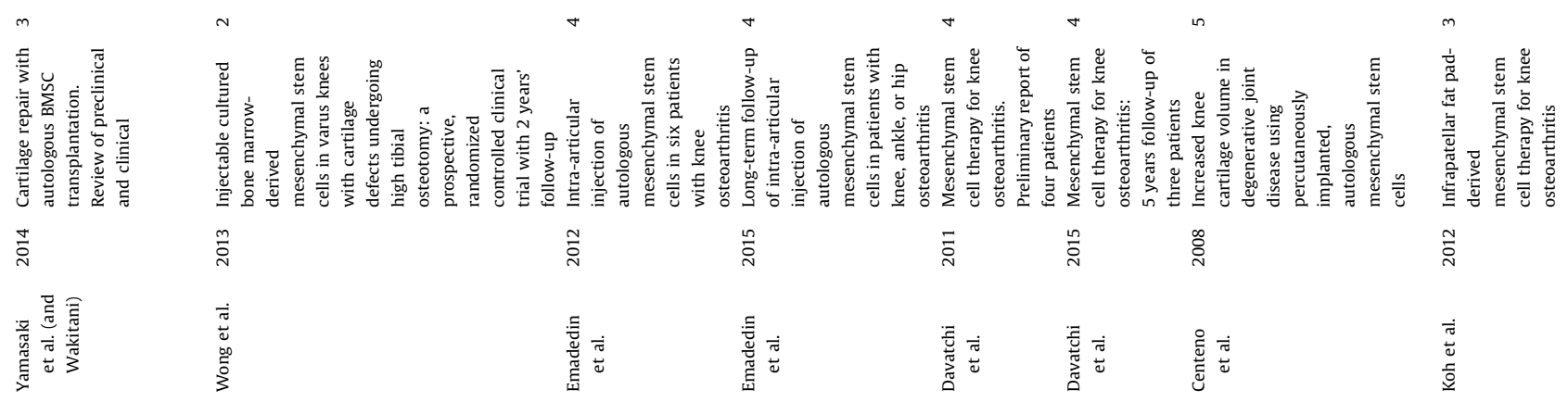




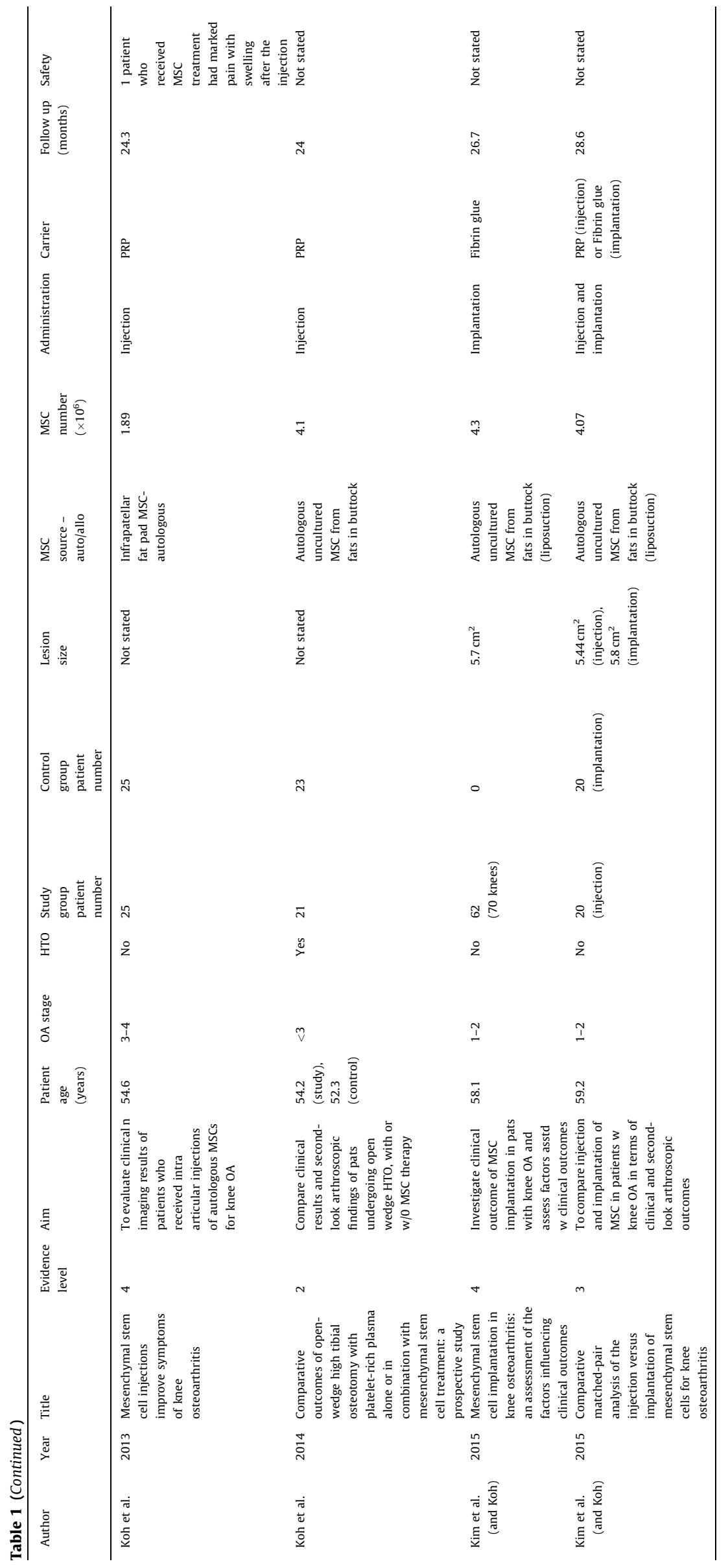


while efficacy of allogeneic treatment was similar to autologous ADSCs, ${ }^{11}$ both were less satisfactory compared to autologous BMSCs. Nevertheless, advantages of using an allogeneic source include lower costs, higher homogeneity, and the added bonus of it being acceptable for use in seropositive patients. ${ }^{1}$

\section{BMSC versus ADSCs, MSC dosage}

The notion that all MSCs are equal is a common misconception. $^{22}$ Significant differences have been found between multipotentiality and function of stem cells derived from different tissues, ${ }^{23,24}$ and even within the same type of tissues sourced from different anatomical sites. ${ }^{25}$ A search through the clinicaltrials.gov website has revealed usage of BMSCs (24 studies), ADSCs (14 studies), and umbilical stem cells (6 studies) to target OA repair. Surprisingly, only BMSCs and ADSCs have been tested in the abovementioned publications. As none of the papers directly compared the efficacy of these 2 MSC sources, it is not possible to draw a conclusion as to which is a superior candidate for OA repair.

MSC dosage is another hypothesized prognostic factor for OA repair. BMSCs have been infused as low as $8 \times 10^{6},{ }^{14}>10 \times 10^{6}, 2,4$ to $>20 \times 10^{6} .9,12$ Markedly better results were observed with BMSCs treatment, ${ }^{1,8-10,12}$ except for a study which infused the lowest number of MSCs at $8 \times 10^{6}$ and attributed the mild improvements to MSC effect. ${ }^{14,15}$ Taken together, it is suggestive that the infusion of $>10 \times 10^{6}$ BMSCs is a requirement to achieve a significantly better repair. Infusions of isolated ADSCs tend to be on the lower end, at $1.89 \times 10^{67}$ to $4 \times 10^{6} \mathrm{MSCs}{ }^{3}$ except for a dosecomparative study which injected a record number of $100 \times 10^{6}$ ADSCs and found that cartilage volume increased gradually till 6 months. Lower dosages of $10 \times 10^{6}$ and $50 \times 10^{6}$ ADSCs were found to be less beneficial and led to inconsistent results. ${ }^{11}$ Unexpected correlations between radiological outcomes and the number of stem cells injected were also observed between cell dosages of lower magnitude. ${ }^{16}$

All the studies above introduced MSCs into the patient as a oneoff procedure. A repeated suggestion arising from the works of these groups are calls that future clinical trials include a multiple administration of MSCs, as it could further improve the efficacy of MSC treatment. ${ }^{3,11,12,14}$ A multi-dosage approach might be more practical for an allogeneic MSC strategy, but will still be applicable for autologous MSCs, provided the gridlock faced in generating sufficient cell numbers is overcome.

\section{Severity of $O A$ lesion and patient age}

$\mathrm{OA}$ is a progressively debilitating disease, which thus entwines it closely to time and patient age. Most of the recruited patients in these studies were diagnosed with stage $2 \mathrm{OA}$. When a clinical trial excluded patients beyond 65 years with severe OA, significant reduction was observed in MRI T2 values up till 12 months. This indicated cartilage regeneration. WOMAC and Lequesne scores also decreased in parallel to VAS scores, which led the authors to conclude that patients with mild to moderate $\mathrm{OA}$ are ideal candidates for MSC therapy. ${ }^{10}$ A similar finding was made by Emadedin et al.; observed improvements went into decline 6 months after MSC treatment in stage 4 OA patients. ${ }^{12}$ Patients with lesion size greater than $5.4 \mathrm{~cm}^{2}$ exhibited significantly worse outcomes in IKDC and Tegner assessments. Multivariate analysis revealed high prognostic value significantly related to patient age and lesion size, indicating a cutoff age of 60 years to ensure MSC efficacy in treating OA. ${ }^{17}$ In another RCT involving patients who underwent treatment for knee cartilage defects of ICRS grade 3-4 lesions, early treatment was concluded to be better. ${ }^{26}$ Taken together, these findings highlight the need that $\mathrm{OA}$ researchers should be equipped with analytical tools for early detection and accurate measurement of disease severity.
Efforts are underway to test the robustness of proteomic technology to understand OA pathophysiology, ${ }^{27}$ and plasma biomarker CCL3 was recently discovered to be useful in detecting pre X-ray defined changes and OA stage. ${ }^{28}$

\section{MSC carriers and mode of infusion}

Both MSC transport medium and infusion mode influence viscosity of the MSC suspension, affect the rate at which cells move into the defect site, and ultimately determine the period that cells stay localized onto the defect site. The optimal carrier would support MSC viability, in addition to enhancing its chondrogenesis. ${ }^{29}$ Both parameters differed in the above mentioned studies, with saline, ${ }^{1,9,10,14}$ serum, ${ }^{2,12}$ natural biomaterial PRP, ${ }^{3,6,7,16,17}$ and hyaluronic acid $(\mathrm{HA})^{2}$ selected for injection procedures. Fibrin glue $^{6}$ and Type I collagen ${ }^{4}$ were the delivery vehicles observed for implanted MSCs.

Of these, the usage of PRP is the most controversial. First documented to be used in Italy in 1970, PRP is derived from autologous blood and is a platelet concentrate known to contain and release growth factors capable of stimulating tissue healing. PRP had been used in conjunction with MSC therapy due to its antiinflammatory effect. ${ }^{30}$ Treatment of OA with PRP alone had been shown to be more effective than saline in providing symptomatic relief in early OA for a period of 6 months, ${ }^{31}$ but its usage should be treated with caution as PRP had been observed to induce more transient reactions compared to hyaluronic acid. ${ }^{32}$ PRP also failed to provide superior clinical improvement in comparison to HA. ${ }^{33}$

Advocates of both infusion modes have confidence in their methods as MSCs can directly differentiate into repair tissue and exert trophic effects through secreted bioactive factors. ${ }^{34} \mathrm{~A}$ matched-pair analysis of arthroscopic outcomes following MSC therapy via implantation or injection indicated significantly greater improvements in the implanted group. Although no significant improvement was noted in the injection group at 28.6 months, ${ }^{6}$ significant correlation was detected between the number of administered MSCs and clinical outcomes. This pattern was present only in the injected group. A possible explanation is that efficacy of MSC injections leverage more on trophic effects, which is less powerful and thus exhibited a dose-dependent improvement. Nonetheless, optimized delivery of stem cells to maximize the reparative effect of each cell is highly sought after, to enhance effectiveness of a low-dosage therapy. This was demonstrated by the success of utilizing $1 \times 10^{5}$ MSCs in treating limb ischemia compared to $1 \times 10^{6}$ MSCs. ${ }^{35}$ Newly-developed cryogels have been shown to be advantageous for chondrogenesis, ${ }^{36}$ resistant against sheer-induced damage and useful in tackling problems such as uncontrolled localization and poor retention. ${ }^{37}$

\section{High tibial osteotomy (HTO)}

HTO is a knee alignment surgery which OA patients undergo to relieve pressure on the knee joint. The concurrent infusion of MSCs through implantation or injection had been carried out. ${ }^{2-4}$ Mild insignificant improvements were observed in cell-treated groups implanted with $10 \times 10^{6}$ BMSCs $^{4}$ and injected with $4 \times 10^{6}$ ADSCs $^{3}$ when patients were reviewed 5 years and 2 years respectively later. In contrast, MSC-treated group injected with $10 \times 10^{6}$ BMSCs was found to perform significantly better than the control group upon adjustment for age and baseline scores at 1 year follow-up; complete coverage of lesions with repair tissue was only apparent in the MSC group. 36\% of MSC-treated lesions were $>50 \%$ covered in contrast to $14 \%$ of control group. ${ }^{2}$ Direct comparison between these 3 studies is not possible due to differences in experimental set-up such as marrow stimulation, MSC carrier, and delivery mode. It could be argued, however, that the promising outcome 
in Wong et al. is transient as assessment was done at an earlier time point. Further follow-ups extending beyond 2 years would enable a more thorough assessment.

\section{Safety}

No serious adverse events were observed in any of the studies involving BMSC infusion. ${ }^{2,8,9,12}$ Minor events ranged from discomfort to swelling. ${ }^{1,13}$ A noteworthy finding is that BMSCs infused through implantation were shown to be without any serious side effects even 11 years later. ${ }^{38}$ Minor side effects were reported to occur at similar frequencies in autologous and allogeneic MSC treatments. ${ }^{1}$ However, one patient infused with a low dose of $1.89 \times 10^{6}$ ADSCs had marked pain with swelling immediately after the procedure. ${ }^{7}$ Minor side effects seemed to occur quite frequently with injections involving high dosage ADSC injections of $10 \times 10^{6}(67 \%), 50 \times 10^{6}(67 \%)$, and $100 \times 10^{6} \operatorname{ADSCs}(42 \%)$. ${ }^{11}$

\section{Limitations}

Acknowledged limitations of these studies include low patient numbers, ${ }^{11}$ unknown optimal MSC number, ${ }^{7}$ short follow-up periods, ${ }^{2,3}$ and retrospectively-collected data. ${ }^{6}$ Notwithstanding these shortcomings, they have shown promising data, which encourages further studies, albeit better-designed ones complete with control arms.

\section{Conclusion}

$\mathrm{OA}$ is an undisputedly complex disease, and is a growing worldwide problem. Assessment of safety is always of paramount interest but focus should now be placed into optimizing MSC potency for OA repair. More basic studies are needed to shore up in-depth knowledge, which in turn would allow investigators to zoom in to more efficacious strategies. Future clinical trials should include direct comparisons between MSC sources, with extended follow-ups; the longer the treatment, the better the outcome.

\section{Conflicts of interest}

The authors have none to declare.

\section{References}

1. Vega A, Martin-Ferrero MA, Del Canto F, et al. Treatment of knee osteoarthritis with allogeneic bone marrow mesenchymal stem cells: a randomized controlled trial. Transplantation. 2015;99(8):1681-1690.

2. Wong KL, Lee KB, Tai BC, Law P, Lee EH, Hui JH. Injectable cultured bone marrowderived mesenchymal stem cells in varus knees with cartilage defects undergoing high tibial osteotomy: a prospective, randomized controlled clinical trial with 2 years' follow-up. Arthroscopy. 2013;29(12):2020-2028.

3. Koh YG, Kwon OR, Kim YS, Choi YJ. Comparative outcomes of open-wedge high tibial osteotomy with platelet-rich plasma alone or in combination with mesenchymal stem cell treatment: a prospective study. Arthroscopy. 2014;30(11):1453-1460.

4. Wakitani S, Imoto K, Yamamoto T, Saito M, Murata N, Yoneda M. Human autologous culture expanded bone marrow mesenchymal cell transplantation for repair of cartilage defects in osteoarthritic knees. Osteoarthr Cartil. 2002;10(3):199-206.

5. Yamasaki S, Mera H, Itokazu M, Hashimoto Y, Wakitani S. Cartilage repair with autologous bone marrow mesenchymal stem cell transplantation: review of preclinical and clinical studies. Cartilage. 2014;5(4):196-202.

6. Kim YS, Kwon OR, Choi YJ, Suh DS, Heo DB, Koh YG. Comparative matched-pair analysis of the injection versus implantation of mesenchymal stem cells for knee osteoarthritis. Am J Sports Med. 2015;43(11):2738-2746.

7. Koh YG, Choi YJ. Infrapatellar fat pad-derived mesenchymal stem cell therapy for knee osteoarthritis. Knee. 2012;19(6):902-907.

8. Orozco L, Munar A, Soler R, et al. Treatment of knee osteoarthritis with autologous mesenchymal stem cells: two-year follow-up results. Transplantation. 2014;97(11): e66-e68.

9. Orozco L, Munar A, Soler R, et al. Treatment of knee osteoarthritis with autologous mesenchymal stem cells: a pilot study. Transplantation. 2013;95(12):1535-1541.

10. Soler R, Orozco L, Munar A, et al. Final results of a phase I-II trial using ex vivo expanded autologous mesenchymal stromal cells for the treatment of osteoarthritis of the knee confirming safety and suggesting cartilage regeneration. Knee. 2016.
11. Jo $\mathrm{CH}$, Lee $\mathrm{YG}$, Shin $\mathrm{WH}$, et al. Intra-articular injection of mesenchymal stem cells for the treatment of osteoarthritis of the knee: a proof-of-concept clinical trial Stem Cells. 2014;32(5):1254-1266.

12. Emadedin M, Aghdami N, Taghiyar L, et al. Intra-articular injection of autologous mesenchymal stem cells in six patients with knee osteoarthritis. Arch Iran Med. 2012;15(7):422-428

13. Emadedin M, Ghorbani Liastani M, Fazeli R, et al. Long-term follow-up of intraarticular injection of autologous mesenchymal stem cells in patients with knee, ankle, or hip osteoarthritis. Arch Iran Med. 2015;18(6):336-344.

14. Davatchi F, Abdollahi BS, Mohyeddin M, Shahram F, Nikbin B. Mesenchymal stem cell therapy for knee osteoarthritis. Preliminary report of four patients. Int J Rheum Dis. 2011;14(2):211-215.

15. Davatchi F, Sadeghi Abdollahi B, Mohyeddin M, Nikbin B. Mesenchymal stem cell therapy for knee osteoarthritis: 5 years follow-up of three patients. Int J Rheum Dis. 2015.

16. Koh YG, Jo SB, Kwon OR, et al. Mesenchymal stem cell injections improve symptoms of knee osteoarthritis. Arthroscopy. 2013;29(4):748-755.

17. Kim YS, Choi YJ, Koh YG. Mesenchymal stem cell implantation in knee osteoarthritis: an assessment of the factors influencing clinical outcomes. Am J Sports Med. 2015;43(9):2293-2301.

18. Centeno CJ, Busse D, Kisiday J, Keohan C, Freeman M, Karli D. Increased knee cartilage volume in degenerative joint disease using percutaneously implanted, autologous mesenchymal stem cells. Pain Phys. 2008;11(3):343-353.

19. Sordi V, Piemonti L. Therapeutic plasticity of stem cells and allograft tolerance. Cytotherapy. 2011;13(6):647-660.

20. Murphy JM, Dixon K, Beck S, Fabian D, Feldman A, Barry F. Reduced chondrogenic and adipogenic activity of mesenchymal stem cells from patients with advanced osteoarthritis. Arthritis Rheum. 2002;46(3):704-713.

21. Alsuhaibani O, Pereira WC, Tareeqanwar M, et al. Infectious disease screening among stem cell transplant donors: an institutional experience in Saudi Arabia. Ann Neurosci. 2015;22(2):81-86.

22. Phinney DG, Sensebe L. Mesenchymal stromal cells: misconceptions and evolving concepts. Cytotherapy. 2013;15(2):140-145.

23. Li CY, Wu XY, Tong JB, et al. Comparative analysis of human mesenchymal stem cells from bone marrow and adipose tissue under xeno-free conditions for cell therapy. Stem Cell Res Ther. 2015;6:55.

24. Winter A, Breit S, Parsch D, et al. Cartilage-like gene expression in differentiated human stem cell spheroids: a comparison of bone marrow-derived and adipose tissue-derived stromal cells. Arthritis Rheum. 2003;48(2):418-429.

25. Lopa S, Colombini A, Stanco D, de Girolamo L, Sansone V, Moretti M. Donormatched mesenchymal stem cells from knee infrapatellar and subcutaneous adipose tissue of osteoarthritic donors display differential chondrogenic and osteogenic commitment. Eur Cell Mater. 2014;27:298-311.

26. Vanlauwe J, Saris DB, Victor J, et al. Five-year outcome of characterized chondrocyte implantation versus microfracture for symptomatic cartilage defects of the knee: early treatment matters. Am J Sports Med. 2011;39(12): 2566-2574.

27. Liao W, Li Z, Wang H, Wang J, Fu Y, Bai X. Proteomic analysis of synovial fluid: insight into the pathogenesis of knee osteoarthritis. Int Orthop. 2013;37(6): 1045-1053.

28. Zhao XY, Yang ZB, Zhang ZJ, et al. CCL3 serves as a potential plasma biomarker in knee degeneration (osteoarthritis). Osteoarthr Cartil. 2015;23(8):1405-1411.

29. Bertolo A, Arcolino F, Capossela S, et al. Growth factors cross-linked to collagen microcarriers promote expansion and chondrogenic differentiation of human mesenchymal stem cells. Tissue Eng A. 2015;21(19-20):2618-2628.

30. Osterman C, McCarthy MB, Cote MP, et al. Platelet-rich plasma increases antiinflammatory markers in a human coculture model for osteoarthritis. Am J Sports Med. 2015;43(6):1474-1484.

31. Patel S, Dhillon MS, Aggarwal S, Marwaha N, Jain A. Treatment with platelet-rich plasma is more effective than placebo for knee osteoarthritis: a prospective, double-blind, randomized trial. Am J Sports Med. 2013;41(2):356-364

32. Riboh JC, Saltzman BM, Yanke AB, Fortier L, Cole BJ. Effect of leukocyte concentration on the efficacy of platelet-rich plasma in the treatment of knee osteoarthritis. Am J Sports Med. 2015.

33. Filardo G, Di Matteo B, Di Martino A, et al. Platelet-rich plasma intra-articular knee injections show no superiority versus viscosupplementation: a randomized controlled trial. Am J Sports Med. 2015;43(7):1575-1582.

34. Caplan AI, Dennis JE. Mesenchymal stem cells as trophic mediators. J Cell Biochem. 2006;98(5):1076-1084

35. Li Y, Liu W, Liu F, et al. Primed 3D injectable microniches enabling low-dosage cell therapy for critical limb ischemia. Proc Natl Acad Sci U S A. 2014;111(37): 13511-13516.

36. Bhat S, Tripathi A, Kumar A. Supermacroprous chitosan-agarose-gelatin cryogels: in vitro characterization and in vivo assessment for cartilage tissue engineering. $J R$ Soc Interface. 2011;8(57):540-554.

37. Liu W, Li Y, Zeng Y, et al. Microcryogels as injectable 3-D cellular microniches for site-directed and augmented cell delivery. Acta Biomater. 2014;10(5):1864-1875

38. Wakitani S, Okabe T, Horibe S, et al. Safety of autologous bone marrow-derived mesenchymal stem cell transplantation for cartilage repair in 41 patients with 45 joints followed for up to 11 years and 5 months. J Tissue Eng Regen Med. 2011;5(2):146-150.

\section{Web reference}

https://clinicaltrials.gov/ct2/results?term=\%22 mesenchymal+stem+

cells\%22+AND+\%22osteoarthritis\%22\&Search=Search.

Search terms: "mesenchymal stem cell" and "osteoarthritis". 